\title{
Single nucleotide polymorphisms in matrix metalloproteinase genes and lung cancer chemotherapy response and prognosis
}

\author{
D.B. Scherf*, H. Dally\#, P. Müller*, G. Werle-Schneider*, B. Jäger*, L. Edler`, \\ S. Tuengerthal ${ }^{+}$, J.R. Fischer ${ }^{\S}$, P. Drings ${ }^{+}$, H. Bartsch" ${ }^{\#}$ and A. Risch*
}

ABSTRACT: The prognosis for lung cancer patients treated with chemotherapy is poor. Single nucleotide polymorphisms (SNPs) in matrix metalloproteinase (MMP) genes could influence treatment outcome by altering apoptotic pathways. Eight SNPs with known or suspected phenotypic effect in six genes (MMP1, MMP2, MMP3, MMP7, MMP9 and MMP12) were investigated.

For 349 Caucasian patients with primary lung cancer, receiving first-line chemotherapy, three different endpoints were analysed: response after the second cycle, progression free survival (PFS) and overall survival (OS).

The prognostic value of the SNPs was analysed using multiple logistic regression for all patients and histology-, stage- and treatment-specific subgroups. Hazard ratio estimates for PFS and OS were calculated using Cox regression methods.

None of the investigated polymorphisms modified response significantly in the whole patient population.

However, tumour stage IIIB variant allele carriers of MMP2 C-735T showed a significantly worse response. PFS was significantly prolonged in MMP1 G-1607GG variant allele carriers and OS in small cell lung cancer patients carrying the MMP12 A-82G variant allele.

In conclusion, this study identified SNPs in MMP1, MMP2, MMP7 and MMP12 for further investigation as possible predictors of chemotherapy outcome in lung cancer patients.

KEYWORDS: Apoptosis, lung cancer chemotherapy, matrix metalloproteinase, pharmacogenetics, polymorphism

W ith about 1.35 million new cases diagnosed per year, lung cancer is the most common cancer worldwide [1]. It is a disease with major morbidity and continuingly bad prognosis. While early stage nonsmall cell lung cancer (NSCLC) can be treated by surgery, late stage NSCLC and small cell lung cancer (SCLC) cases receive chemotherapy as the treatment of choice [2]. Polymorphisms, which reduce or inhibit apoptosis, can cause chemotherapy resistance [3]. Cohort studies are used in order to elucidate correlations between biomarkers in the host genome, shown to be relevant for patient outcome, and therapy response.

Matrix metalloproteinase (MMP) expression is associated with the development of an extensive list of diseases especially various malignant tumours. Their involvement in promotion of metastasis, chemotherapy resistance of cancer and a bad treatment outcome has been shown in previous studies $[4,5]$.

High expression of MMPs is usually associated with all steps of cancer initiation and progression. MMP3, MMP7 and MMP9, have also been reported to influence the Fas/FasL-mediated extrinsic, as well as the p53/PKC mediated intrinsic apoptotic pathway [6, 7]. By cleavage of plasminogen and collagen XVIII, MMP12 is one of the most effective producers of the angiogenesis inhibitors angiostatin and endostatin [8]. In addition, it has an influence on the plasmin levels and subsequent activation of MMPs like MMP3 and MMP9 [9]. MMP2, also known as gelatinase $\mathrm{A}$, is involved in migration and invasion processes and also seems to have influence on chemotherapy response. It has been shown in rat models that platinum-based chemotherapy has higher response rates when the

\section{AFFILIATIONS}

Depts of *Epigenomics and Cancer Risk Factors,

"Toxicology and Cancer Risk

Factors, and

"Biostatistics, German Cancer

Research Center,

+Thoraxklinik Heidelberg, Heidelberg, and

${ }^{\S}$ Klinik Löwenstein, Löwenstein,

Germany.

CORRESPONDENCE

A. Risch

Dept of Epigenomics and Cancer Risk Factors

German Cancer Research Center Heidelberg

Germany

E-mail: a.risch@dkfz.de

Received:

Aug 142008

Accepted after revision:

July 132009

First published online:

July 302009
European Respiratory Journal Print ISSN 0903-1936 Online ISSN 1399-3003 
animals are co-treated with prinomastat, a specific MMP2 inhibitor [10]. MMP1 is the most highly expressed interstitial collagenase, degrading fibrillar collagen. Overexpression of MMP1 shown in tumour tissues has been suggested to be associated with tumour invasion and metastasis [11].

Polymorphisms in MMP genes have been shown to influence the expression pattern of the protein. One polymorphism in the promoter region of $M M P 2(C-735 T)$ was reported to lead to a lower expression of the protein due to a disruption of a SP1binding site [12]. As far as $M M P 3$ is concerned, the variant allele of the ins/del polymorphism $(6 A-11715 A)$ is associated with a higher transcription rate [13]. For $M M P 7$, two single nucleotide polymorphisms (SNPs) (C-153T and $A-181 G)$ have been functionally characterised and, in both cases, the variant alleles lead to an enhanced expression [14]. Another SNP in the promoter of the MMP9 gene (C-1562T) increases transcription by the disruption of a repressor binding site [12]. Polymorphisms in the MMP12 gene $(A-82 G ; A 1082 G)$ also have been shown to influence the outcome of cancer patients. The promoter polymorphism is responsible for lowering the transcription by disruption of an AP1-binding site [15], whereas the polymorphism $A 1082 G$ has not been shown to have a phenotypic effect. The GG allele of the functional MMP1 G-1607GG polymorphism is associated with higher expression levels of MMP1 and with increased susceptibility to head and neck and lung cancer $[16,17]$.

This study focuses on the relationship between polymorphisms in MMP genes and three defined endpoints of clinical outcome: 1) overall response rate (ORR) to chemotherapy after the second cycle; 2) overall survival (OS); and 3) progression free survival (PFS). We hypothesised that genetic background, such as polymorphisms in gDNA can influence therapy outcome. We also assumed that this background has a more immediate effect on an early endpoint, such as ORR, than the clinically more relevant late endpoints, OS and PFS, due to tumour-specific accumulation of genetic variations by clonal selection during many cycles of therapy.

Eight SNPs in six MMP genes were analysed in a cohort of 349 lung cancer patients, consisting of 187 NSCLC, 161 SCLC and one patient with a mixed histology, receiving first-line chemotherapy.

\section{PATIENTS AND METHODS}

\section{Study cohort}

349 patients of Caucasian origin with histologically confirmed primary lung cancer eligible for first-line chemotherapy were recruited between March 1999 and October 2004 at the Thoraxklinik in Heidelberg. The cut-off date for the followup was March 2005. All patients had never received antineoplastic chemotherapy nor had they previously been diagnosed with another malignancy. Where possible, tumour stage at the time of diagnosis was determined according to the clinical tumour, node, metastasis (cTNM) of the Union Internationale contre le Cancer [18] using hospital records. For some SCLC patients, the tumour stage was classified as limited or extensive disease based on the Veterans Administration Lung Cancer Study Group criteria [19]. For statistical analysis, limited and extensive disease were regarded as stage III and IV, respectively (table 1).

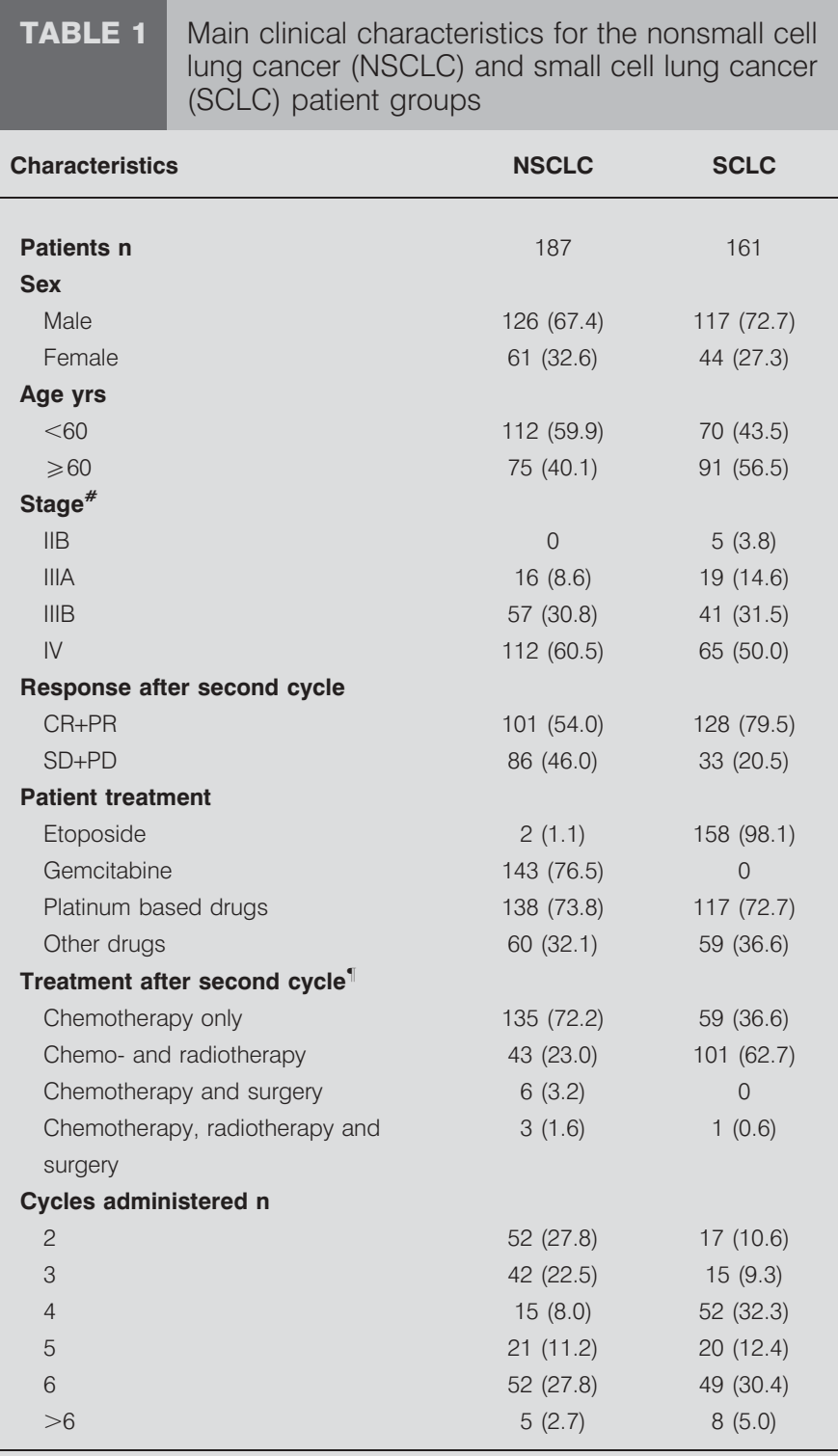

Data are presented as $n(\%)$, unless otherwise stated. One patient with mixed histology is not included in this table. CR: complete remission; PR: partial remission; SD: stable disease; PD: progressive disease. ${ }^{*}$ : for two NSCLC patients $(1.1 \%)$, stage was unknown. For 31 SCLC patients, the tumour stage was only classified as limited or extensive disease based on the Veterans Administration Lung Cancer Study Group criteria. Limited and extensive disease were regarded as stage II-III and IV, respectively. ": patients with surgery or radiotherapy prior to the second cycle of chemotherapy were not included in the study.

Tumour response was assessed after the second cycle of firstline chemotherapy as complete remission (CR), partial remission (PR), stable disease (SD) or progressive disease (PD) according to RECIST (response evaluation criteria in solid tumours) [20]. Progression free survival (PFS) is defined as the time interval (in days) between start of chemotherapy and documented progression (uncensored observation). In case no progression was documented, PFS was calculated until the last progression-free examination (censored observation) 
irrespective of whether that patient was lost to follow up or whether death occurred later. OS was defined as the time interval (in days) between start of chemotherapy and the documented date of death (uncensored observation) or, when date of death was unknown, the last date when the patient was still alive (censored observation).

$60 \%$ of the 187 NSCLC cases died during the observation period the median OS time was 410 days (95\% CI 353-514). The median PFS time was 216 days (95\% CI 189-251), and the median observation time was 812 days (95\% CI 499-1120). 63\% of the 161 SCLC patients died during observation, the median OS time was 405 days (95\% CI 347-557). The median PFS time was 271 days (95\% CI 239-314), and the median observation time was 777 days (95\% CI 648-838). All participants signed informed consent forms and most study subjects completed a self-administered questionnaire with detailed information on pre-treatment, smoking habits and occupational exposure. The study was approved by the ethical committee of the Medical Faculty of the University of Heidelberg (Heidelberg, Germany) (Ref.-Nr.199/ 2001). Blood samples were collected prior to chemotherapy.

\section{DNA extraction}

Buffy coat from $5 \mathrm{~mL}$ venous blood in EDTA was archived at $-80^{\circ} \mathrm{C}$. Genomic DNA was isolated using either the QIAamp DNA blood midi kit (Qiagen, Hilden, Germany), or by an automated DNA extraction protocol on the MagNA Pure System (Roche, Basel, Switzerland) according to the manufacturer's instructions.

\section{Genotyping}

For the detection of the polymorphisms MMP3 6A-1171 $5 A$, MMP12 A-82G and MMP12 A1082G, assays using fluorescencebased melting curve analysis (LightCycler480) were designed (shown in table 2). Each PCR with a final volume of $10 \mu \mathrm{L}$ was performed in 96-well plates (Roche, Mannheim, Germany) with $\sim 20 \mathrm{ng}$ of genomic DNA as template. After $2 \mathrm{~min}$ of $95^{\circ} \mathrm{C}$ initial incubation, 45 subsequent cycles of $5 \mathrm{~s}$ at $95^{\circ} \mathrm{C}, 10 \mathrm{~s}$ for annealing at $56^{\circ} \mathrm{C}$ and $15 \mathrm{~s}$ at $72^{\circ} \mathrm{C}$ for elongation were carried out. Concentrations for the multiplex reaction for MMP12 A-82G and MMP12 A1082G genotyping were $1 \times$ PCR Buffer, $5 \mathrm{mM} \mathrm{MgCl}_{2}$,
$200 \mu \mathrm{M}$ dNTPs, $0.5 \mathrm{U}$ Qiagen Polymerase, $0.7 \mu \mathrm{M}$ forward primer $(A-82 \mathrm{G}), 0.2 \mu \mathrm{M}$ reverse primer $(A-82 \mathrm{G}), 0.1 \mu \mathrm{M}$ forward primer $(A 1082 G), 0.7 \mu \mathrm{M}$ reverse primer $(A 1082 G)$ and for all four probes $0.2 \mu \mathrm{M}$ each. Concentrations for MMP3 6A-1171 5A genotyping were $0.1 \mu \mathrm{M}$ forward primer and $0.5 \mu \mathrm{M}$ reverse primer, $2.5 \mathrm{mM} \mathrm{MgCl} 2,1 \times$ PCR buffer, $200 \mu \mathrm{M}$ dNTPs, $0.5 \mathrm{U}$ Taq Polymerase (Eurolone, England) and both probes $0.2 \mu \mathrm{M}$ each. All primers and probes (TIB MOLBIOL, Berlin, Germany) are detailed in table 2 .

For validation and quality control of the LightCycler480 method, PCR restriction fragment length polymorphism (RFLP) was performed for MMP12 A-82G [21]. Another PCR-RFLP analysis, for MMP12 A1082G was newly developed and carried out in a total volume of $20 \mu \mathrm{L}$ with $400 \mathrm{nM}$ of each primer (forward primer 5'-GATGACAAATACTGGTTAATTAGGA-3'; reverse primer 5'-CTGGTTATCTACAAAGAAGT-3'), $200 \mu \mathrm{M}$ dNTPs, $1 \times$ PCR Buffer, $0.8 \mathrm{U}$ Taq-Polymerase and $5 \mathrm{mM}$ magnesium. Cycling conditions were as follows: initial denaturation at $95^{\circ} \mathrm{C}$ for $3 \mathrm{~min}$ followed by 35 cycles of denaturation at $94^{\circ} \mathrm{C}$ for $30 \mathrm{~s}$, annealing at $59^{\circ} \mathrm{C}$ for $30 \mathrm{~s}$, elongation at $72^{\circ} \mathrm{C}$ for $30 \mathrm{~s}$ with a temperature transition rate of $20^{\circ} \mathrm{C}$ per second and a final elongation step at $72^{\circ} \mathrm{C}$ for $2 \mathrm{~min}$. Results were compared for at least 200 samples and showed 100\% concordance. All genotyping was carried out blinded before data analysis. $10 \%$ of samples were genotyped twice for quality control and results showed no discrepancies. PCR-RFLP methods were employed as previously published for MMP1 G-1607GG, MMP3 6A-1171 5A, MMP2 C735T, MMP9 C-1562T, MMP7 C-153T and MMP7 A-181G [12, 14, $17,22]$.

Linkage analysis of the polymorphisms situated on the long arm of chromosome 11 was calculated using the HAPReg software. Calculation of haplotypes and the logistic regression for evaluation of haplotypic effect was conducted with the Thesias software [23].

\section{Statistical analysis}

The allele frequencies and their standard deviation for the group of all patients were calculated and the genotype distribution was tested for deviations from Hardy-Weinberg

\section{TABLE 2 Primers and probes}

\begin{tabular}{|c|c|c|c|}
\hline Gene & Primers/probes & Primer sequence $\left(5^{\prime}-3^{\prime}\right)$ & Annealing temperature ${ }^{\circ} \mathrm{C}$ \\
\hline MMP12 & Forward & TGCTAATTGATCCATTGTCG/ & 57 \\
\hline \multirow[t]{2}{*}{ A-82G } & Reverse & GAGCTCCAGAAGCAGTGG/ & 57 \\
\hline & Sensor mut & 610-TGAATCCTATGAGTGACTCACAGTTGAT-PH & \\
\hline MMP12 & Forward & TGGGAACCATAGAAAAGAGACTA/ & 57 \\
\hline \multirow[t]{2}{*}{ A1082G } & Reverse & GGTCCTATAAAAACGTGGGT/ & 57 \\
\hline & Sensor wt & ACTGGTTAATTAGCAATTTAAGACCAG-FL & \\
\hline MMP3 & Forward & GAGCTGCCACAGCTTCTACA/ & 55 \\
\hline \multirow[t]{3}{*}{$6 \mathrm{~A}-11715 \mathrm{~A}$} & Reverse & CTCAACCTCTCAAAGTGCTAGGAT/ & 55 \\
\hline & Anchor & 640-CCATCAAAGGAATGGAGAACCATAGAATAC & \\
\hline & Sensor wt & AAGACATGGTTTTTTTCCCCX & \\
\hline
\end{tabular}


equilibrium (HWE) using the Chi-squared test. The prognostic value of the SNPs was analysed using multivariable logistic regression. Odds ratios were calculated by comparing genotype frequencies in responders (CR and $P R$ ) and nonresponders (SD and $\mathrm{PD}$ ) after the second cycle of first-line chemotherapy.

Hazard ratio (HR) estimates with 95\% confidence intervals were calculated using Cox proportional hazard models for PFS (adjusted for tumour stage) and for OS (adjusted for tumour stage and sex). Kaplan-Meier survival curves were plotted and the log-rank test was used to test for differences in both PFS and OS. In univariate analysis, tumour stage had a statistically significant influence on the response after the second cycle of NSCLC patients $(p=0.0012)$, and on PFS and OS of all lung cancer patients $(p<0.001)$ in this study. Therefore, we included stage as adjustment factor in all analyses. Sex had a significant influence on OS $(p=0.044)$ but not on PFS $(p=0.982)$ of all lung cancer patients and also not on chemotherapy response. Therefore, it was included as an adjustment factor only for OS. There was no significant influence of age in all three clinical outcomes (for response $p=0.59$, for OS $p=0.88$, and for PFS $\mathrm{p}=0.82$ ). The same was observed for performance status measured by ECOG (for response $p=0.61$, for OS $p=0.81$, and for PFS $p=0.13$ ). Thus, neither of these two parameters was used as confounding factor.
This analysis was performed for all 349 lung cancer patients. In addition, it was performed separately for the two histological sub-populations of SCLC and NSCLC. The wild-type genotype was considered as reference. We analysed therapy-based subsets including the patients receiving gemcitabine- (143; all NSCLC), etoposide- (161; 158 SCLC, 2 NSCLC and 1 mixed) and platinum-based therapy (256; 117 SCLC and 139 NSCLC). Additionally we analysed early- (IIB+IIIA) and late-stage $(\mathrm{IIIB}+\mathrm{IV})$ patients as well as IIIB and IV separately in the histological subgroups.

Allele frequencies were calculated using the website of the International Helmholtz Gemeinschaft [24]. All other calculations were performed using the statistical software package SAS version 9.1.3 (SAS Institute, Cary, NC, USA).

\section{RESULTS}

The total of 349 lung cancer patients, who received first-line chemotherapy were included into this study. Three different endpoints were analysed: response after the second cycle (R), PFS and OS, each for the whole patient group, as well as for the two subgroups, SCLC and NSCLC.

The distribution of the genotypes of the SNPs analysed is given in table 3. For all SNPs, the distribution of genotypes was within Hardy-Weinberg equilibrium (MMP1 G-1607GG p $=0.65$

TABLE 3 Association of matrix metalloproteinase (MMP) single nucleotide polymorphisms and the response after the second cycle of chemotherapy for all lung cancer patients

\begin{tabular}{|c|c|c|c|c|c|c|c|c|}
\hline Polymorphism & Genotypes & Patients n & $\begin{array}{c}\text { All } \\
(n=349)\end{array}$ & $\begin{array}{c}\text { SCLC } \\
(n=161)\end{array}$ & $\begin{array}{l}\text { NSCLC } \\
(n=187)\end{array}$ & $\begin{array}{l}\text { Stage IIB+IIIA } \\
\qquad(n=40)\end{array}$ & $\begin{array}{c}\text { Stage IIIB } \\
\qquad(n=98)\end{array}$ & $\begin{array}{l}\text { Stage IV } \\
(n=177)\end{array}$ \\
\hline \multicolumn{9}{|l|}{ MMP1 } \\
\hline \multirow[t]{3}{*}{ G-1607GG } & $\mathbf{G} / \mathbf{G}$ & 96 & 1 (ref) & 1 (ref) & 1 (ref) & 1 (ref) & 1 (ref) & 1 (ref) \\
\hline & G/GG+ & 253 & $0.9(0.5-1.4)$ & $0.9(0.3-2.1)$ & $1.0(0.5-1.9)$ & $0.65(0.13-3.31)$ & $1.03(0.37-2.83)$ & $0.8(0.4-1.4)$ \\
\hline & $\mathrm{GG} / \mathrm{GG}$ & & & & & & & \\
\hline \multicolumn{9}{|l|}{ MMP2 } \\
\hline \multirow[t]{2}{*}{ C-735T } & CC & 266 & 1 (ref) & 1 (ref) & 1 (ref) & 1 (ref) & 1 (ref) & 1 (ref) \\
\hline & $\mathrm{CT}+\mathrm{TT}$ & 81 & $1.5(0.9-2.6)$ & $2.1(0.9-4.8)$ & $1.5(0.7-3.0)$ & $6.0(1.1-31.3)^{\#}$ & $2.8(1.1-7.4)^{9}$ & $1.0(0.5-2.1)$ \\
\hline \multicolumn{9}{|l|}{ MMP3 } \\
\hline \multirow[t]{2}{*}{$6 A-11715 A$} & $6 \mathrm{~A} 6 \mathrm{~A}$ & 90 & 1 (ref) & 1 (ref) & 1 (ref) & 1 (ref) & 1 (ref) & 1 (ref) \\
\hline & $6 A 5 A+5 A 5 A$ & 259 & $1.4(0.8-2.4)$ & $1.4(0.6-3.4)$ & $1.2(0.6-2.3)$ & $1.1(0.20-8.48)$ & $10.4(1.3-81.5)^{+}$ & $0.9(0.5-1.6)$ \\
\hline \multicolumn{9}{|l|}{ MMP7 } \\
\hline \multirow[t]{2}{*}{ C-153T } & CC & 287 & 1 (ref) & 1 (ref) & 1 (ref) & 1 (ref) & 1 (ref) & 1 (ref) \\
\hline & $\mathrm{CT}+\mathrm{TT}$ & 60 & $1.8(1.0-3.2)$ & $2.2(0.9-5.4)$ & $1.7(0.8-3.9)$ & $6.0(1.1-31.3)^{\S}$ & $1.54(0.52-4.70)$ & $1.4(0.6-2.9)$ \\
\hline \multirow[t]{2}{*}{$A-181 G$} & AA & 66 & 1 (ref) & 1 (ref) & 1 (ref) & 1 (ref) & 1 (ref) & 1 (ref) \\
\hline & $A G+G G$ & 283 & $1.0(0.6-1.8)$ & $1.0(0.4-2.6)$ & $1.1(0.5-2.2)$ & $1.3(0.1-14.1)$ & $1.39(0.41-4.62)$ & $0.7(0.3-1.5)$ \\
\hline \multicolumn{9}{|l|}{ MMP9 } \\
\hline \multirow[t]{2}{*}{ C-1562T } & CC & 261 & 1 (ref) & 1 (ref) & 1 (ref) & 1 (ref) & 1 (ref) & 1 (ref) \\
\hline & $\mathrm{CT}+\mathrm{TT}$ & 87 & $0.9(0.5-1.5)$ & $1.2(0.5-2.8)$ & $0.7(0.4-1.4)$ & $2.0(0.4-10.8)$ & $0.6(0.1-2.1)$ & $1.0(0.5-1.9)$ \\
\hline \multicolumn{9}{|l|}{ MMP12 } \\
\hline \multirow[t]{2}{*}{$A-82 G$} & AA & 54 & 1 (ref) & 1 (ref) & 1 (ref) & 1 (ref) & 1 (ref) & 1 (ref) \\
\hline & $A G+G G$ & 94 & $0.9(0.5-1.5)$ & $0.8(0.3-1.8)$ & $1.3(0.7-2.7)$ & $0.9(0.2-5.3)$ & $1.0(0.3-2.6)$ & $0.8(0.4-1.5)$ \\
\hline \multirow[t]{2}{*}{ A1082G } & AA & 308 & 1 (ref) & 1 (ref) & 1 (ref) & 1 (ref) & 1 (ref) & 1 (ref) \\
\hline & $A G+G G$ & 40 & $0.7(0.3-1.4)$ & $0.4(0.1-1.7)$ & $1.0(0.4-2.7)$ & $0.5(0.0-4.1)$ & N/A & $1.1(0.5-2.7)$ \\
\hline
\end{tabular}

Data presented as OR (95\% Cl), unless otherwise stated. Genotype results are missing for MMP2 C-735T, MMP7 C-153T (two patients each), MMP9 C-1562T, MMP12 A82G, MMP12 A1082G (one patient each). ORs were adjusted for stage. SCLC: small cell lung cancer; NSCLC: nonsmall cell lung cancer; N/A: not applicable. Bold indicates significance. ${ }^{\#}: p=0.03 ;{ }^{\bullet}: p=0.02 ;{ }^{+}: p=0.01 ;^{\S}: p=0.03$. 


\begin{tabular}{|c|c|c|c|c|c|c|c|c|c|}
\hline \multirow[t]{2}{*}{ Polymorphism } & \multirow[t]{2}{*}{ Genotypes } & \multicolumn{2}{|c|}{ Stage IIB+IIIA } & \multicolumn{2}{|c|}{ Stage IIIB+IV } & \multicolumn{2}{|c|}{ Stage IIIB } & \multicolumn{2}{|c|}{ Stage IV } \\
\hline & & Patients $\mathrm{n}$ & Response & Patients n & Response & Patients $\mathrm{n}$ & Response & Patients n & Response \\
\hline \multicolumn{10}{|l|}{ MMP1 } \\
\hline \multicolumn{10}{|l|}{ MMP2 } \\
\hline \multirow[t]{2}{*}{ C-735T } & CC & 19 & 1 (ref) & 77 & 1 (ref) & 27 & 1 (ref) & 50 & 1 (ref) \\
\hline & $\mathrm{CT}+\mathrm{TT}$ & 5 & $12.8(1.4-67.4)^{\#}$ & 27 & $2.1(0.8-5.5)$ & 13 & $2.6(0.5-12.5)$ & 14 & $2.0(0.5-7.0$ \\
\hline \multicolumn{10}{|l|}{ MMP3 } \\
\hline C-153T & $\mathrm{CT}+\mathrm{TT}$ & 4 & $5.7(0.6-57.2)$ & 21 & $1.5(0.5-4.3)$ & 6 & $0.8(0.1-8.0)$ & 15 & $1.7(0.5-6.1)$ \\
\hline \multirow[t]{2}{*}{ A-181G } & AA & 3 & 1 (ref) & 16 & 1 (ref) & 6 & 1 (ref) & 10 & 1 (ref) \\
\hline & $A G+G G$ & 21 & $0.5(0.0-6.6)$ & 90 & $0.9(0.3-3.0)$ & 35 & $1.25(0.1-12.5)$ & 55 & $0.7(0.2-3.2$ \\
\hline \multicolumn{10}{|l|}{ MMP9 } \\
\hline \multirow[t]{2}{*}{ C-1562T } & CC & 20 & 1 (ref) & 82 & 1 (ref) & 35 & 1 (ref) & 47 & 1 (ref) \\
\hline & $\mathrm{CT}+\mathrm{TT}$ & 4 & $5.7(0.5-66.7)$ & 24 & $1.6(0.5-4.3)$ & 6 & $0.8(0.0-6.1)$ & 18 & $1.9(0.5-6.1)$ \\
\hline \multicolumn{10}{|l|}{ MMP12 } \\
\hline \multirow[t]{2}{*}{ A-82G } & AA & 20 & 1 (ref) & 68 & 1 (ref) & 29 & 1 (ref) & 39 & 1 (ref) \\
\hline & $A G+G G$ & 3 & $2.0(0.1-28.0)$ & 38 & $0.5(0.2-1.5)$ & 12 & $0.8(0.1-4.5)$ & 26 & $0.4(0.1-1.5$ \\
\hline \multirow[t]{2}{*}{ A1082G } & AA & 21 & 1 (ref) & 94 & 1 (ref) & 37 & 1 (ref) & 57 & 1 (ref) \\
\hline & $A G+G G$ & 2 & $<0.1(<0.0-999)$ & 12 & $0.7(0.0-2.7)$ & 4 & $<0.1(<0.0-999)$ & 8 & $1.0(0.2-5.7)$ \\
\hline
\end{tabular}

Data are presented as crude OR $(95 \% \mathrm{Cl})$, unless otherwise stated. N/A: not applicable. Bold indicates significance. ${ }^{\#}: \mathrm{p}=0.03$.

MMP2 C-735T $\mathrm{p}=0.57 ; M M P 36 A-11715 A \mathrm{p}=0.39 ; M M P 7 \mathrm{C}-153 \mathrm{~T}$ $\mathrm{p}=0.11 ; A-181 \mathrm{G} \mathrm{p}=0.18 ; M M P 9 C-1562 T \mathrm{p}=0.71 ; M M P 12 A-82 \mathrm{G}$ $\mathrm{p}=0.97 ;$ and $A 1082 \mathrm{G} \mathrm{p}=0.99)$. The calculated frequencies of the wild-type alleles were MMP2 C-735T 0.88 $\pm 0.015, M M P 36 A$ $11715 A \quad 0.49 \pm 0.024, \quad M M P 7 \quad C-153 T \quad 0.93 \pm 0.012, \quad A-181 G$ $0.46 \pm 0.022, \quad M M P 9 \quad C-1562 T \quad 0.86 \pm 0.016, \quad M M P 12 \quad A-82 G$ $0.85 \pm 0.017$, and $A 1082 G 0.93 \pm 0.012$. These frequencies are comparable to those previously published for Caucasians by other groups [5, 12-14].

While for SCLC, chemotherapy is the treatment of choice for all stages, early stage NSCLC patients are preferentially treated surgically. This is reflected in table 1 where response rates of SCLC patients are also much higher compared to NSCLC patients.

Odds ratios and their 95\% confidence intervals describing the risk of being a nonresponder for variant allele carriers after multivariate analysis are shown in table 3.

In the group of all lung cancer patients, none of the examined polymorphisms significantly modified the chemotherapy response after the second cycle; only a borderline significant effect was observed for the MMP7 C-153T polymorphism (OR $1.79,95 \%$ CI $0.99-3.20 ; p=0.05)$. Among stage IIIB patients, the risk of being a nonresponder was significantly increased in the aggregated groups of variant allele homozygotes and heterozygotes for MMP2 C-735T (OR 2.79, 95\% CI 1.06-7.41; $\mathrm{p}=0.02$ ) and MMP3 6A-1171 5A (OR 10.37, 95\% CI 1.32-81.46; $\mathrm{p}=0.01)$ (table 3).

An enhanced risk for being a nonresponder was also found for IIB-IIIA patients among the SCLC cohort carrying the MMP2 C-735T polymorphism (OR 12.75, 95\% CI 1.39-167.39; $\mathrm{p}=0.03$ ) as well as for stage IIIB NSCLC patients carrying the variant allele of the MMP3 $6 A-11715 A$ polymorphism (OR 7.78, 95\% CI 1.35-147.84; $\mathrm{p}=0.03$ ) (tables 4 and 5).

PFS was significantly affected in the group of NSCLC patients, who received gemcitabine as first-line chemotherapeutic drug and are carriers of the variant allele of the MMP12 A1082G polymorphism (HR 1.88, 95\% CI 1.02-3.46; $\mathrm{p}=0.04$ ). Individuals with the variant genotype of MMP9 C-1562T in the whole cohort among late stage patients (HR 1.36, 95\% CI 1.00$1.84 ; \mathrm{p}=0.05$, data not shown) and also among late-stage SCLC patients (adjusted HR 1.72, 95\% CI 1.02-2.89; p=0.04) (table 6) had a significant shorter PFS than those with the wild-type genotype. The Kaplan-Meier curve describing the progressionfree survival for all patients also shows a significant modulation by the MMP9 polymorphism (fig. 1). Individuals in the whole cohort carrying the GG allele of the MMP1 G-1607GG allele had a significantly longer PFS compared with the reference genotype carriers (HR 0.72, 95\% CI 0.55-0.95; $\mathrm{p}=0.04$ ) and a longer PFS and OS in early stage SCLC (tables 6 and 7). 


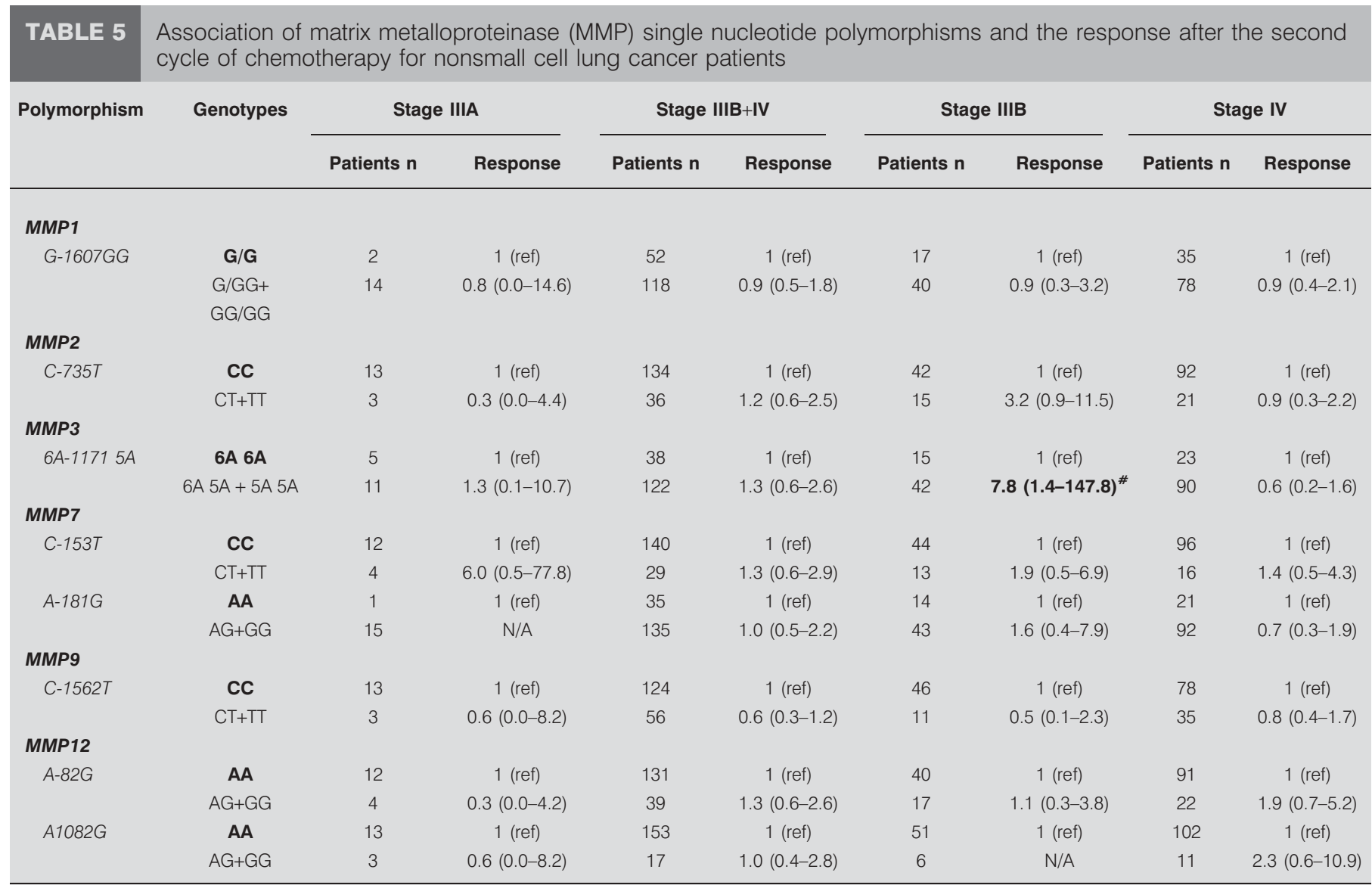

Data are presented as crude OR $(95 \% \mathrm{Cl})$, unless otherwise stated. N/A: not applicable. Bold indicates significance. ${ }^{*}: \mathrm{p}=0.03$.

\section{DISCUSSION}

The choice of therapy for the treatment of lung cancer therapy is currently dependent on stage, histology and performance status of the patient. Given that there are a number of chemotherapy treatments to choose from, host genetic polymorphisms which affect therapy success should be taken into account. Genotyping prior to therapy would be feasible because it is a quick, reliable and minimally invasive procedure as only a blood sample is required. Individually tailored treatment could improve patient survival and quality of life and is thus very desirable for the future.

Therefore, we genotyped seven polymorphisms in five different matrix metalloproteinase genes in a hypothesis-driven approach and analysed their impact on three endpoints: chemotherapy response after the second cycle, PFS and OS. Response rates in SCLC and NSCLC are known to differ strongly and are also dependent on therapy received. Therefore, we stratified data for histology, therapy protocol and stage. Stage has been shown to correlate strongly with MMP expression.

A particular strength of this study lies in the analysis of three endpoints, chemotherapy response, PFS and OS. Numbers are adequate for the separate analysis of SCLC and NSCLC. A big limitation for this study is the lack of adequate sample numbers in the stage subgroups. This is illustrated by the fact that certain ORs and HRs are not calculable for the MMP3 polymorphism in the SCLC cohort due to a lack of wild-type carriers in the nonresponder group. Small sample sizes in stage subgroups also lead some to inconsistencies in the results between the whole cohort and the histological subgroups; e.g. the nonsignificant point estimate for chemotherapy response for carriers of the MMP2 variant allele points towards a protective effect among NSCLC stage IIIA patients, but is associated with a significant risk of nonresponse among all Stage IIB and IIIA and Stage IIIB patients. However a number of effects appear consistent, if not significant, across subgroups.

MMP1 G-1607GG variant allele carriers have a longer PFS than individuals homozygous for the $G$ allele. This result is not in concordance with the hypothesis that the GG allele, representing higher MMP1 expression, has harmful effects during therapy and in the later endpoints. We observed a significant protective effect in the whole cohort and especially in early stage SCLC. This result has to be validated in further studies of preferably larger cohorts.

The variant allele of $M M P 2$ correlates significantly with a poorer response in IIIB and SCLC early-stage patients and the variant allele of $M M P 3$ correlates significantly, with a poorer response in IIIB patients, especially NSCLC, but not stage IV patients after the second cycle of chemotherapy.

Regarding $M M P 2$, the result does not reflect the hypothesised benefit of the $\mathrm{T}$ allele, which is expected to result in low 


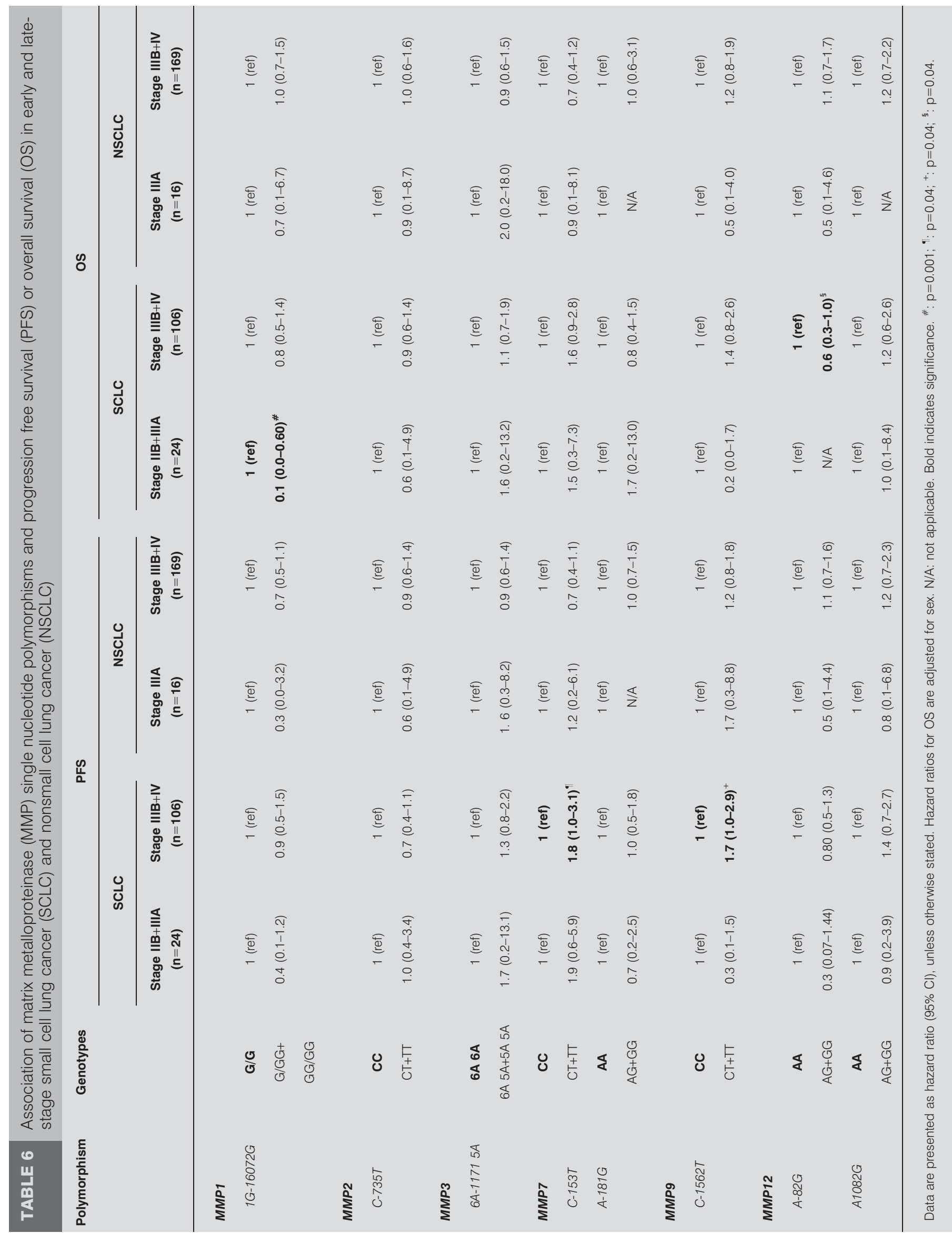



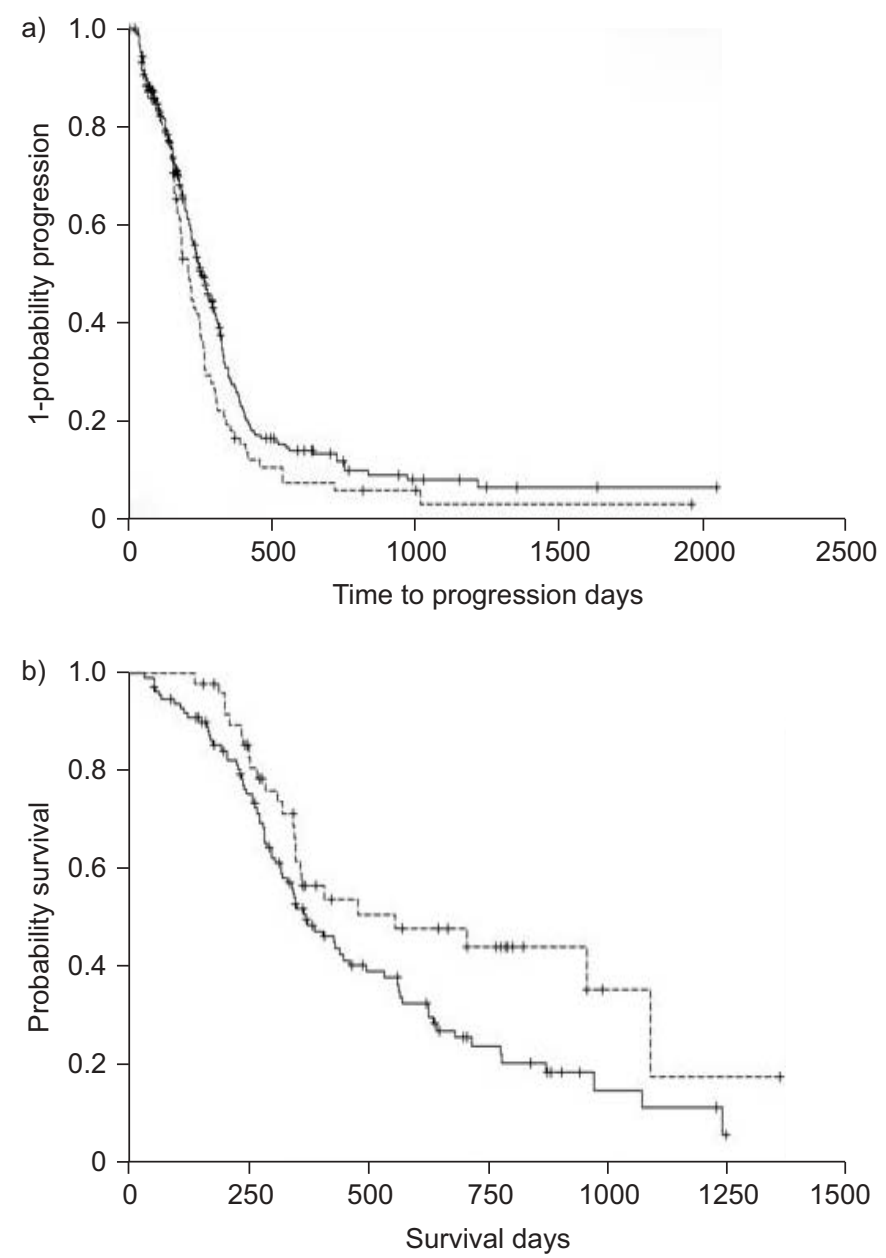

FIGURE 1. Selected Kaplan-Meier curves showing different patient groups in relation to their genotypes for matrix metalloproteinase (MMP) 9 and the promoter polymorphisms of MMP12. a) MMP9 C-1562T, progression free survival of all patients $(n=349)$. — : CC; - - -: TT+CT. $p=0.0460$. b) MMP12 A-82G, overall survival in small cell lung cancer patients $(n=161)$. — : AA; - - -: $G G+A G$. $p=0.0321$.

expression. This is not in accordance with LIU et al. [10], in which rats treated with platinum-based therapy in combination with prinomastat, a specific MMP2 inhibitor, had a higher response. Why this effect is seen in the logistic regression analysis is not clear and should be confirmed in other studies.

The significant results for MMP3 and MMP7 show large confidence intervals, which means the point estimate of the effect size is much less precise. However, they are in accordance with a publication by BLONS et al. [6] which suggests modulation of chemotherapy response by altered MMP3 expression. The reason for this could be enhanced Fas ligand cleavage from the cell surface by MMP7 and MMP3 [25], leading to inhibition of the extrinsic apoptotic pathway.

A possible explanation for why this effect, in the case of the MMP7C-153T polymorphism is observed only in early-stage patients is that the genotype-dependent modulation of treatment outcome is expected to be stronger before extensive metastasis. Both MMP3 and MMP7 are well known for their involvement in metastasis. In stage IV patients where metastasis has already occurred, other resistance mechanisms which are not specific for MMP3 and MMP7 could be more important for response after the second cycle of chemotherapy. The results presented here, which will require confirmation in further pharmacogenetic studies, suggest a genotype specific modulation of therapy protocol in earlier stages of lung cancer.

The Kaplan-Meier estimate for all lung cancer patients shows a significant harmful effect of the $\mathrm{T}$ allele, situated in the promoter region of MMP9 and representing high expression [26], on the progression free survival. This is in accordance with previous publications, which have shown high MMP9 expression as relevant to metastasis and patient outcome. However, this result could not be confirmed by corresponding hazard ratios for the MMP9 C-1562T analysis.

The MMP7 A-181G polymorphism did not show a synergetic effect with $C-153 T$ in the logistic regression. SCLC patients carrying the variant allele even have a longer lifetime after starting chemotherapy. This is not in accordance with the assumption, that both polymorphisms have similar effects on the transcription [14]. However, haplotypic analysis shows a harmful effect of the GT haplotype, which is due to a combination of the high expression alleles. The reason for this is not clear and further studies are required to validate this result.

Due to the antiangiogenic features of MMP12 [27] it was predicted that the hypothetically low expressing $G$ allele has a harmful effect on overall survival and progression free survival. This could not be confirmed by our data. In contrast, it showed a significant protective tendency in the analysis for the overall survival for SCLC patients.

Low expression level also represents a higher plasmin level and a consequently higher activation rate of MMP9 in a MMP3 dependent manner, which might promote tumour growth and shorten PFS and survival [28]. Several studies indicate a benefit of MMP12 expression in various cancers [8, 29]. However, it was also shown that NSCLC patients who have high MMP12 expression have earlier relapse [30]. A reason for this effect could be that there is a link between high MMP12 expression, especially in the macrophages in the lung [31], COPD, inflammation and cancer progression [32-34] and that a lower expression indicates a better outcome for the patients. This would also indicate a tissue dependency especially in men, where cases of COPD appear more frequently.

The function of the exon polymorphism of MMP12 is still unknown, but in this study it associated with bad outcome for NSCLC patients who received gemcitabine as chemotherapy. An explanation for this effect remains speculative but a change in the amino acid sequence of the hemopexin-like domain could alter its affinity or specificity.

Of course, the overall survival and the PFS are parameters which are most clinically relevant. However, in the long run, studies which measure the effect of genotypes on chemotherapy response could, if the results are confirmed by larger independent study cohorts, contribute to better treatment protocols. To our knowledge, this is the first pharmacogenetic study on MMPs and lung cancer that complements analysis of tumour progression and patient survival with an evaluation of the early tumour response. The results prioritise MMP1, 


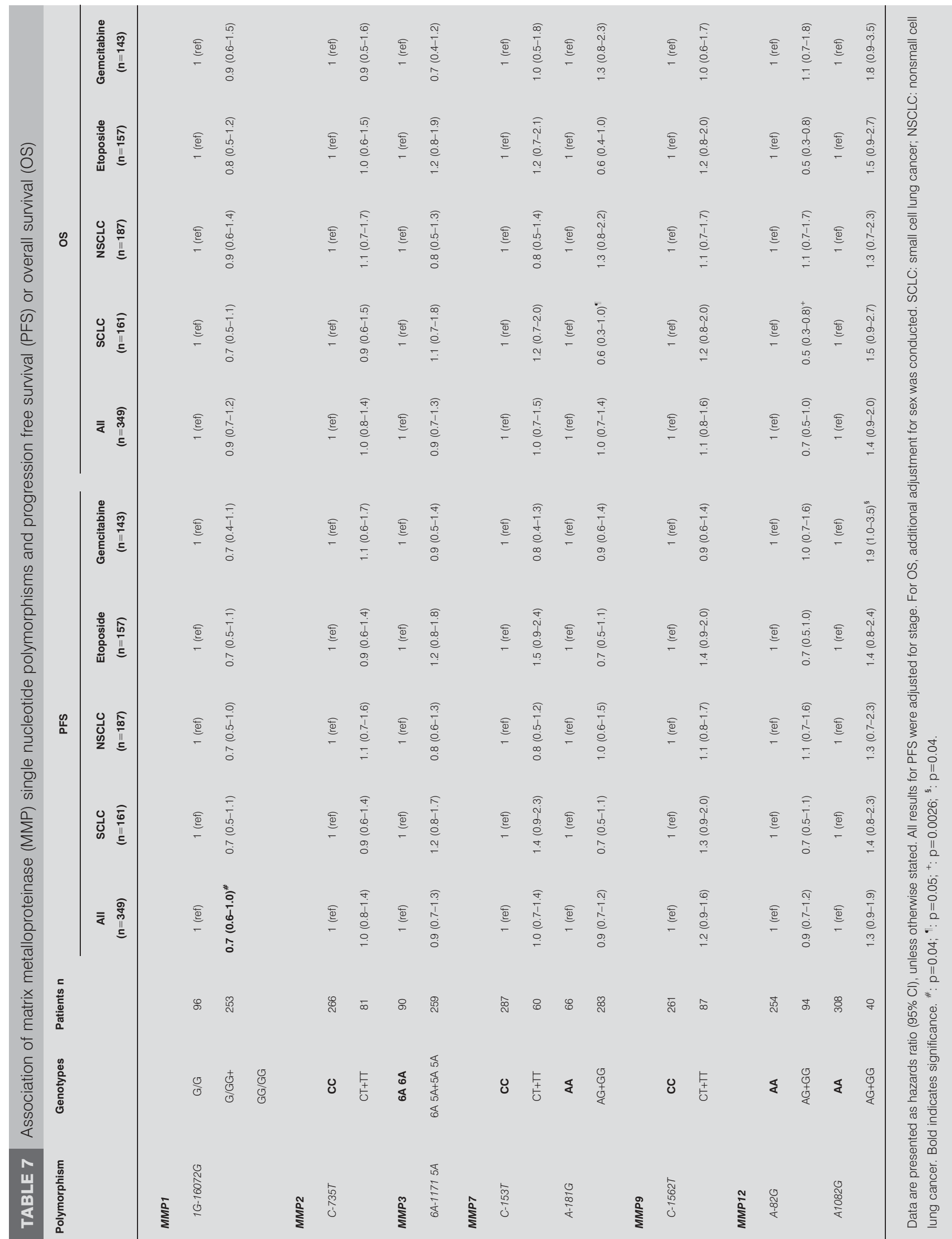


MMP2, MMP7, MMP9 and MMP12 as candidate genes to be further investigated as possible predictors of the clinical outcome of chemotherapy in lung cancer patients.

\section{SUPPORT STATEMENT}

This work was supported, in part, by the Cooperation Program in Cancer Research of the Deutsches Krebsforschungszentrum (DKFZ), by the "Deutsche Krebshilfe", and by the "Verein zur Förderung der Krebsforschung in Deutschland e. V."

\section{STATEMENT OF INTEREST}

None declared.

\section{ACKNOWLEDGEMENTS}

The authors are grateful to all patients and staff at the Thoraxklinik $\mathrm{GmbH}$ (Heidelberg, Germany) who helped to make this study possible. They also thank those who were involved with sample and/or data collection and archiving, O. Landt (TIB MOLBIOL, Berlin, Germany) for helping with the LightCycler probe design, R. Rausch (Dept of Biostatistics, German Cancer Research Center, Heidelberg) for assisting with statistical analysis as well as M. Timofeeva and L. Knoefel (both Dept of Epigenomics and Cancer Risk Factors, German Cancer Research Center, Heidelberg) for valuable discussions.

\section{REFERENCES}

1 Parkin DM, Bray F, Ferlay J, et al. Global cancer statistics, 2002. CA Cancer J Clin 2005; 55: 74-108.

2 Van Cleave JH, Cooley ME. Lung cancer. In: Van Cleave JH, Cooley ME, eds. A Cancer Sourcebook for Nurses. Sudbury, MA, Jones and Bartlett Publishers Inc., 2004; pp. 215-229.

3 Igney FH, Krammer PH. Death and anti-death: tumour resistance to apoptosis. Nat Rev Cancer 2002; 2: 277-288.

4 Overall $\mathrm{CM}$, Kleifeld O. Tumour microenvironment - opinion: validating matrix metalloproteinases as drug targets and antitargets for cancer therapy. Nat Rev Cancer 2006; 6: 227-239.

5 Heist RS, Marshall AL, Liu G, et al. Matrix metalloproteinase polymorphisms and survival in stage I non-small cell lung cancer. Clin Cancer Res 2006; 12: 5448-5453.

6 Blons H, Gad S, Zinzindohoue F, et al. Matrix metalloproteinase 3 polymorphism: a predictive factor of response to neoadjuvant chemotherapy in head and neck squamous cell carcinoma. Clin Cancer Res 2004; 10: 2594-2599.

7 Meyer E, Vollmer JY, Bovey R, et al. Matrix metalloproteinases 9 and 10 inhibit protein kinase C-potentiated, p53-mediated apoptosis. Cancer Res 2005; 65: 4261-4272.

8 Gorrin Rivas MJ, Arii S, Furutani M, et al. Expression of human macrophage metalloelastase gene in hepatocellular carcinoma: correlation with angiostatin generation and its clinical significance. Hepatology 1998; 28: 986-993.

9 Hahn-Dantona E, Ramos-DeSimone N, Sipley J, et al. Activation of proMMP-9 by a plasmin/MMP-3 cascade in a tumor cell model. Regulation by tissue inhibitors of metalloproteinases. Ann $N$ Y Acad Sci 1999; 878: 372-387.

10 Liu J, Tsao MS, Pagura M, et al. Early combined treatment with carboplatin and the MMP inhibitor, prinomastat, prolongs survival and reduces systemic metastasis in an aggressive orthotopic lung cancer model. Lung Cancer 2003; 42: 335-344.

11 Sauter W, Rosenberger A, Beckmann L, et al. Matrix metalloproteinase 1 (MMP1) is associated with early-onset lung cancer. Cancer Epidemiol Biomarkers Prev 2008; 17: 1127-1135.

12 Rollin J, Regina S, Vourc'h P, et al. Influence of MMP-2 and MMP-9 promoter polymorphisms on gene expression and clinical outcome of non-small cell lung cancer. Lung Cancer 2007; 56: 273-280.

$13 \mathrm{Su}$ L, Zhou W, Asomaning K, et al. Genotypes and haplotypes of matrix metalloproteinase 1,3 and 12 genes and the risk of lung cancer. Carcinogenesis 2006; 27: 1024-1029.
14 Jormsjo S, Whatling $\mathrm{C}$, Walter $\mathrm{DH}$, et al. Allele-specific regulation of matrix metalloproteinase-7 promoter activity is associated with coronary artery luminal dimensions among hypercholesterolemic patients. Arterioscler Thromb Vasc Biol 2001; 21: 1834-1839.

15 Shin A, Cai Q, Shu XO, et al. Genetic polymorphisms in the matrix metalloproteinase 12 gene (MMP12) and breast cancer risk and survival: the Shanghai Breast Cancer Study. Breast Cancer Res 2005; 7: R506-R512.

16 charoenrat $\mathrm{P}$, Leksrisakul $\mathrm{P}$, Sangruchi S. A functional polymorphism in the matrix metalloproteinase-1 gene promoter is associated with susceptibility and aggressiveness of head and neck cancer. Int J Cancer 2006; 118: 2548-2553.

17 Zhu Y, Spitz MR, Lei L, et al. A single nucleotide polymorphism in the matrix metalloproteinase- 1 promoter enhances lung cancer susceptibility. Cancer Res 2001; 61: 7825-7829.

18 Bülzebruck H, Bopp R, Drings P, et al. New aspects in the staging of lung cancer. Prospective validation of the International Union Against Cancer TNM classification. Cancer 1992; 70: 1102-1110.

19 Zelen M. Keynote address on biostatistics and data retrieval. Cancer Chemother Rep 3 1973; 4: 31-42.

20 Therasse P, Arbuck SG, Eisenhauer EA, et al. New guidelines to evaluate the response to treatment in solid tumors. European Organization for Research and Treatment of Cancer, National Cancer Institute of the United States, National Cancer Institute of Canada. J Natl Cancer Inst 2000; 92: 205-216.

21 Jormsjo S, Ye S, Moritz J, et al. Allele-specific regulation of matrix metalloproteinase- 12 gene activity is associated with coronary artery luminal dimensions in diabetic patients with manifest coronary artery disease. Circ Res 2000; 86: 998-1003.

22 Dunleavey L, BeyzadeS, YeS. Rapid genotype analysis of the stromelysin gene 5A/6A polymorphism. Atherosclerosis 2000; 151: 587-589.

23 THESIAS Software Version $3.1 \mathrm{http}: / /$ ecgene.net/genecanvas/ uploads/THESIAS3.1/Documentation3.1.htm Date last updated: 2004. Date last accessed: 2008.

24 IHG. Hardy-Weinberg Equilibrium (HWE). http://ihg2 helmholtz-muenchen.de/cgi-bin/hw/hwa1.pl Date last accessed: October 2008.

25 Matsuno H, Yudoh K, Watanabe Y, et al. Stromelysin-1 (MMP-3) in synovial fluid of patients with rheumatoid arthritis has potential to cleave membrane bound Fas ligand. J Rheumatol 2001; 28: 22-28.

26 Zhang B, Ye S, Herrmann SM, et al. Functional polymorphism in the regulatory region of gelatinase $B$ gene in relation to severity of coronary atherosclerosis. Circulation 1999; 99: 1788-1794.

27 Galligioni E, Ferro A. Angiogenesis and antiangiogenic agents in non-small cell lung cancer. Lung Cancer 2001; 34: Suppl. 4, S3-S7.

28 Inuzuka K, Ogata $\mathrm{Y}$, Nagase $\mathrm{H}$, et al. Significance of coexpression of urokinase-type plasminogen activator, and matrix metalloproteinase 3 (stromelysin) and 9 (gelatinase B) in colorectal carcinoma. J Surg Res 2000; 93: 211-218.

29 Houghton AM, Grisolano JL, Baumann ML, et al. Macrophage elastase (matrix metalloproteinase-12) suppresses growth of lung metastases. Cancer Res 2006; 66: 6149-6155.

30 Hofmann HS, Hansen G, Richter G, et al. Matrix metalloproteinase-12 expression correlates with local recurrence and metastatic disease in non-small cell lung cancer patients. Clin Cancer Res 2005; 11: 1086-1092.

31 Nenan S, Boichot E, Lagente V, et al. Macrophage elastase (MMP12): a pro-inflammatory mediator? Mem Inst Oswaldo Cruz 2005; 100: Suppl. 1, 167-172.

32 Mannino DM. COPD and lung cancer have come a long way...baby. Am J Respir Crit Care Med 2007; 176: 108-109.

33 Abidi P, Afaq F, Arif JM, et al. Chrysotile-mediated imbalance in the glutathione redox system in the development of pulmonary injury. Toxicol Lett 1999; 106: 31-39.

34 Han MK, Postma D, Mannino D, et al. Gender and COPD: why it matters. Am J Respir Crit Care Med 2007; 176: 1179-1184. 